\title{
Dual-polarized UWB Antenna Array
}

\author{
Grzegorz Adamiuk, Student Member, IEEE, Malgorzata Janson, Student Member, IEEE, Werner \\ Wiesbeck, Fellow, IEEE, and Thomas Zwick, Senior Member, IEEE
}

\begin{abstract}
The paper describes a dual-polarized antenna array for Impulse Radio Ultra Wideband (IR-UWB) applications. Firstly a brief description of the single radiator is given. Next the prototype is presented and measured data is compared with the simulated one. The linear array consisting of four components achieves a very narrow mean $3 \mathrm{~dB}$ beam width, which is approx. $15^{\circ}$ in the $\mathbf{F C C}$ frequency range from $3.1 \mathrm{GHz}$ to $10.6 \mathrm{GHz}$. The second main lobe of the array factor and the grating lobes are suppressed by the radiation pattern of the single element. The mean cross-polarization suppression in the main beam direction is larger than $20 \mathrm{~dB}$. Next the simulated and measured time domain radiation properties of the antenna array are presented. The array is applicable in the polarimetric, impulse based ultra wideband systems with high angular selectivity and high range resolution.
\end{abstract}

Index Terms-Antennas, Arrays, UWB, polarization diversity.

\section{INTRODUCTION}

One of the remarkable advantages of the Impulse Radio Ultra Wideband (IR-UWB) system is a very large bandwidth. In the regulation defined originally by FCC [1] it covers the frequencies from $3.1 \mathrm{GHz}$ to $10.6 \mathrm{GHz}$. Such bandwidth allows for a very fine resolution in e.g. Radar [3], where the range resolution increases directly with the increasing bandwidth. However there is a limited number of the antenna types, which are able to radiate over the relative bandwidth of approx. $100 \%$. Additionaly for the operation in IR-UWB there are several additional requirements on the antenna, which have to be fulfilled, when working with very short pulses. One of them is geometrical constancy of the phase center of radiation in the considered frequency range. The variation of the phase center distorts the pulse, what decreases the possible resolution.

Since many objects scatter the electromagnetic wave differently for the different polarizations, it is possible to increase the performance of the radar or imaging system by an application of a polarization diversity [5]. For this reason dualorthogonaly polarized UWB antennas are needed. The main requirements for the antennas are the sufficient impedance matching, the proper radiation pattern for the intended application, the phase center of radiation, which is constant over frequency and is the same for both polarizations. For a polarimetry a good polarization decoupling in a far-field is required. In the radar the directive antennas with a small beamwidth are desired. In the literature there are presented only few candidates for such antennas [6], [7], [8], so there exist still a research need on the antennas for IR-UWB. For an

The Authors are with the Institut fuer Hochfrequenztechnik und Elektronik, Universitaet Karlsruhe (TH), Kaiserstr. 12, 76131, Karlsruhe, Germany, email:grzegorz.adamiuk@ihe.uka.de increase of the angular resolution the antenna arrays introduce a solution [4]. The array should radiate with one main beam and low grating lobes over the desired bandwidth. For this reason small and possibly directive antennas are needed. Since the literature does not introduce many solutions for the given task, in this paper the dual-polarized antenna array for the IRUWB with small beamwidth and high polarization purity is presented.

The paper is arranged in the following way. In the section II the single array element is briefly introduced. In the section III a simulation model of the radiation from the antenna array is described. In the section IV the results obtained from the measurements and simulations are shown for the frequency and the time domain. Finally the conclusions are given.

\section{Single RADiATOR}

The dual-polarized antenna element is manufactured in planar technology. It consists of an array of broadband monopoles. The elements are surrounded by a ground plane formed to a circle as shown in Fig. 1. To achieve a single linear polarization two oppositely placed monopoles have to be excited simultaneously. In order to excite a desired mode in the antenna the monopoles have to be excited with out-ofphase signals. In order to obtain the vertical polarization the orientation of the electric field vectors at the feeding points has to be as indicated by the arrows in Fig. 1. For the horizontal polarization the two orthogonal monopoles have to be excited analogously to the described scheme.

Due to the symmetry of the antenna around the center point the eqivalent planes for both polarizations maintain the same radiation properties. The phase center of radiation for the both polarizations is in the same geometrical point, which is the middle of the structure. An equal phase center for both polarizations is important in order to guarantee the same radiation conditions for both polarizations. The phase center of radiation does not change its position over the frequency. This is vital for the IR-UWB systems.

In one half-space of the antenna the feeding networks are placed. Both, feeding networks and antenna are etched on the subtrate Duroid 5880 with the relative permittivity $\epsilon_{r}=2.2$ and the thickness $h=0.79 \mathrm{~mm}$. The input signal is fed to the microstrip line. Next the signal is split with a $3 \mathrm{~dB}$ - power divider. The microstrip lines are tapered to the modified slot lines which are directly soldered to the feeding points of the single monopoles. The layouts of the feeding networks are shown in Fig. 2. The both feeding networks must be crossed in order to feed orthogonal polarizations. This would result in an intersection of the power dividers. For this reason a vertical disalignment of the power dividers must have been 
performed. The feeding networks are soldered perpendicularly to the antenna surface.

Since the radiation of the antenna is symmetrical to the antenna surface, the feeding network is directly illuminated by the radiated signal. It results in a reflection and scattering of the signal, which generate contributions in an uncontrollable polarization. In order to overcome the problem the part of the energy radiated in the direction of the feeding network is absorbed. A photo of the resulting antenna is shown in Fig. 3 , where four elements are arranged in an array.

In order to evaluate the antenna properties the transfer function $H_{A n t}(t, \theta, \psi)$ of the device must be measured. For this purpose the transmission $S_{21}(f, \theta, \psi)$ from the antenna under test AUT to the reference antenna in an anechoic chamber was measured with a VNA. The network analyser was calibrated with the reference points beeing the inputs of the AUT and the reference antenna. The measured transmission factor $S_{21}(f, \theta, \psi)$ is a product of the transfer function of the antenna under test $H_{A n t}(f, \theta, \psi), j \omega$, free space propagation and of the transfer function of the reference antenna $H_{\text {ref }}(f, \theta, \psi)$.

$$
S_{21}(f, \theta, \psi)=H_{A n t}(f, \theta, \psi) \cdot \frac{j \omega e^{-\frac{j \omega r}{c_{0}}}}{2 \pi r c_{0}} \cdot H_{r e f}(f, \theta, \psi)
$$

With the formula $H_{A n t}(f, \theta, \psi)$ can be obtained. From the complex transfer function all radiation properties of the antenna can be derived. The gain $G(f, \theta, \psi)$ is calculated with the following formula [9], [10]

$$
G(f, \theta, \psi)=\frac{4 \pi f^{2}}{c^{2}}|H(f, \theta, \psi)|^{2}
$$

The radiation pattern $C(f, \theta, \psi)$ is calculated as follows

$$
C\left(f_{0}, \theta, \psi\right)=\frac{H\left(f_{0}, \theta, \psi\right)}{H_{\max }\left(f_{0}, \theta, \psi\right)}
$$

The gain $G$ in the H-plane over frequency $f$ is shown in Fig. 4(b). The radiation pattern of the antenna is very stable over the frequency and is directive. No sidelobes are present. The characteristic is nerly symmetrical w.r.t. the main beam direction.

For the IR-UWB systems the impulse response $h(t, \theta, \psi)$ is a quantity that described the transient behavior of the antenna. It is expected that is as short as possible and shows no oscillations after the main pulse. The impulse response can be obtained by the Fourier transform of the transfer function $H(f, \theta, \psi)[11]$.

$$
h(t, \theta, \psi)=\mathcal{F}^{-1}\{H(f, \theta, \psi)\}
$$

The impulse response of the antenna $h_{A n t}(f, \theta, \psi)$ is shown in Fig. 7(a). The length of the impulse response is very short, which yields the small distortion of the radiatied signal. Short length of the pulse is a result of the high stability of the phase center of radiation over the frequency. Some weak ringing after the main pulse is present. It is a result of the reflection of the signal from the feeding network, which could not be absorbed. The delay caused by the antenna is very stable over

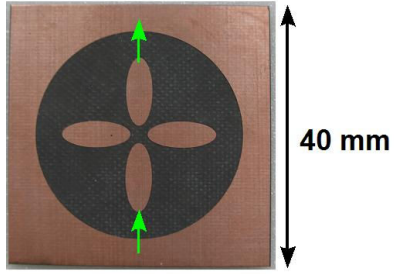

Fig. 1. Photo of the radiating section of the dual-polarized UWB antenna.

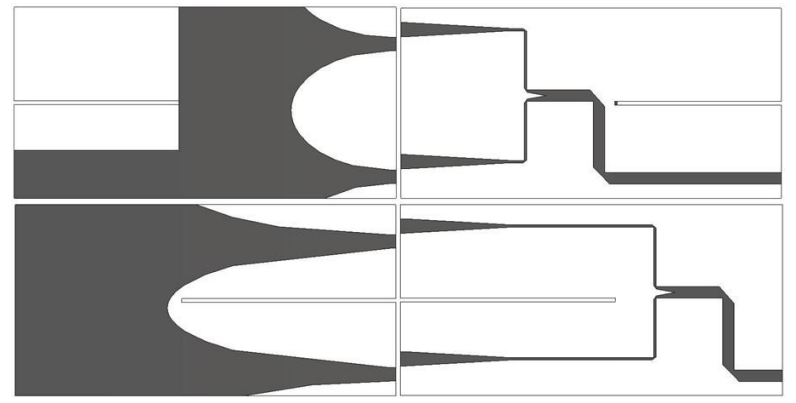

Fig. 2. Layout of the feeding networks for both polarizations. (up - top and bottom view of the feeding network for vertical polarization; down - top and bottom view of the feeding network for horizontal polarization)

the angle $\theta$, which confirms the high stability of the phase center of radiation over the geometrical dimensions.

The measurements for the orthogonal polarization show very similar behavior. The only differences are in the slightly different gain, due to the different arrangement of the feeding network for both polarizations. For this reason in the following the results only for one polarization are introduced.

\section{RADiATION PATTERN OF AN UWB ANTENNA ARRAY}

The arrays, that are considered here are linear arrays with equal excitation at each element. In order to calculate the radiation pattern of an array the following input data must be specified:

- transfer function of the single element $H_{A n t}(f, \theta, \psi)$

- number of elements in the array $N$

- distance between the elements $d$

- complex transfer function of the feeding network (power divider and connecting cables) $H_{\text {feed }}(f)$

\section{A. Array Factor}

The complex normalized array factor is calculated by the following formula [2]:

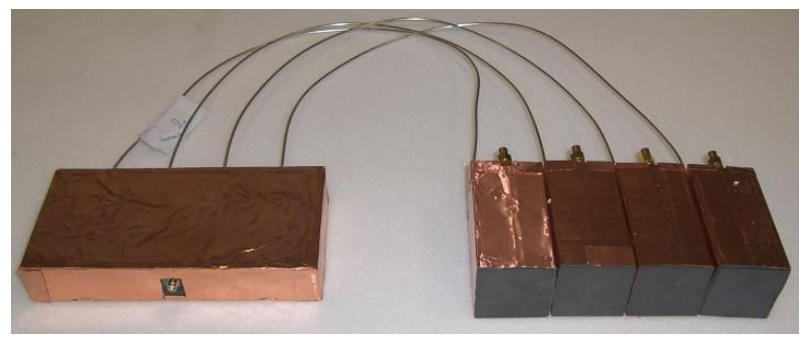

Fig. 3. Photo of the UWB $4 \times 1$ Antenna array with power divider. 


$$
\begin{array}{r}
A F(f, \theta, \psi)=\sum_{i=-\frac{N-1}{2}}^{\frac{N-1}{2}} \frac{1}{\sqrt{N}} \cdot e^{-j \beta_{0}\left(r_{i}-r_{0}\right)} \\
=\sum_{i=-\frac{N-1}{2}}^{\frac{N-1}{2}} \frac{1}{\sqrt{N}} \cdot e^{-j \beta_{0} i d \cos (\theta) \sin (\psi)},
\end{array}
$$

where $r_{i}$ is the distance of the ith element to the observation point in the far field, $r_{0}$ is the distance of the center of the array to the same observation point in the far field, $N$ is number of elements in the array, $d$ is the distance between the elements, $\beta_{0}=2 \pi / \lambda_{0}=2 \pi f / c_{0}, \lambda_{0}$ is the free space wavelength, $f$ is the frequency and $\theta, \psi$ are the azimuth and elevation angles, respectively.

The here considered antenna array is a one dimensional linear antenna array and for this reason only one dimensional array factor will be considered $\left(\psi=90^{\circ}\right)$. The amplitude distribution of the array factor $A F\left(f, \theta, \psi=90^{\circ}\right)$ over frequency is shown in Fig. 4(b). The four element array and the distance $d$ between the elements of $40 \mathrm{~mm}$ is assumed. The distance $d$ is equal to the transversal dimension of the single antenna and is mandatory the minimal distance between the elements in the array.

The resulting transfer function of the array $H_{a r}(f, \theta, \psi)$ is a multiplication of the array factor $A F(f, \theta, \psi)$ and the transfer function of the single element $H_{A n t}(f, \theta, \psi)$. If the antenna has a directive radiation pattern it is possible to supress the main lobe in the direction $180^{\circ}$ and the grating lobes. This allows for the operation with single, narrow lobe. The resulting radiation pattern includung the effects of the feeding network is shown in Fig. 4(c) and discussed in the next section.

\section{B. Transfer function of the feeding network}

The here considered feeding network consist of the $4 \times 1$ power divider and the connecting cables. The signal propagating through the feeding network experiences a delay and attenuation. The losses and the delay can be included in the simulation by the capturing of the complex $S_{x 1}(f)$ parameters, where $x(=2,3,4,5)$ stands for the one of the four outputs of the feeding network and $x=1$ is the input of the power divider.

The transfer function of the feeding network $H_{\text {feed }}(f)$ is

$$
H_{\text {feed }}(f)=S_{x 1} * \sqrt{N},
$$

where $S_{x 1}$ stands for the S-parameter between the input and xth output, $N$ stands for the number of outputs. It describes the overall available amplitude and phase at the outputs of the feeding network. It models the losses in the feeding network, as well as the delay caused by it. In the simulation the reflections at the interface feeding network - antennas are neglected.

\section{Transfer function of the array}

The transfer function of the antenna array is calculated with the following formula:

$$
\begin{array}{r}
H_{a r}\left(f, \theta, \psi=90^{\circ}\right)= \\
H_{\text {Ant }}\left(f, \theta, \psi=90^{\circ}\right) \cdot A F\left(f, \theta, \psi=90^{\circ}\right) \cdot H_{\text {feed }}(f) .
\end{array}
$$

$H_{a r}\left(f, \theta, \psi=90^{\circ}\right)$ is complex and characterizes fully the radiation from the antenna array in the considered frequency range for the specified direction. From this parameter both the frequency as well as time domain radiation parameters can be derived (see sectionII.

The power divider was etched on the subtrate Duroid 5880, thickness $h=0.78 \mathrm{~mm}$, relative dielectric constant $\epsilon_{r}=2.2$. The connecting cables are Sucoform 86 cables with the propagation velocity $v_{r}$ beeing $71 \%$ of the velocity of light. To prevent a radiation from the power divider, it was covered with an absorber and metallic sheet. The photo of the prototype is shown in Fig. 3.

\section{RESULTS}

\section{A. Frequency domain}

The simulated and measured frequency domain results of the antenna array are presented in this section. In Fig. 4(a) the measured gain of the single element is presented. In the Fig. 4(b) the array factor calculated accordingly to the formula (5) is shown. The Fig. 4(c) shows the simulated gain of the array calculated with (7). For the comparison the measured gain of the array is presented in Fig. 4(d).

The array radiates with a narrow beam. The beamwidth gets narrower with the increasing frequency. The mean $3 \mathrm{~dB}$ beamwidth is approx. $15^{\circ}$. The gain in the main beam direction is relatively constant. The second main lobe was suppressed by the pattern of the single element. The grating lobes are also attenuated and only at higher frequencies some stronger radiation outside the main beam is present.

In the polarimetric systems a polarization decoupling is crucial. It is expected, that the polarization decupling in the farfield of the antenna is as high as possible. The antenna array has very low cross-polarization components, which are presented in Fig. 5. Comparing to Fig. 4(d) it can be noticed that the cross-polarization is suppressed w.r.t. to the co-polarization by approx. $20 \mathrm{~dB}$ (note the scale). Only at higher frequencies some stronger unsymmetrical radiation occurs. It is due to the stronger cross-polarized radiation of the single element in this region. However since in the IR-UWB the whole bandwith is occupied by the pulse at the same time during radiation, this does not introduce an important drawback for the array characteristics.

For pulse radiating antennas a one single value characterizing an amplitude for the given direction is advantageous. Such quantity is e.g. the mean gain $G_{m}$. It is an arithmetical mean of the gain over the given frequency range in the specified direction.

$$
G_{m}(\theta, \psi)=\frac{1}{f_{2}-f_{1}} \int_{f_{1}}^{f_{2}} G(f, \theta, \psi) d f
$$

The measured and simulated mean gain $G_{m}$ for the co and cross-polarization in the H-Plane is shown in Fig. 6. The maximal value of the mean gain reaches approx. $7.2 \mathrm{dBi}$. This 


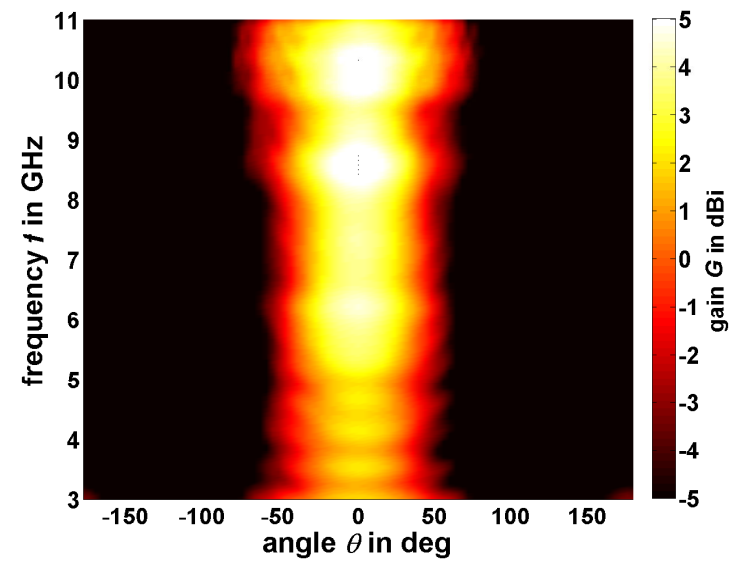

(a) Measured gain $G$ of the single array element over angle $\theta$ and frequency $f$.

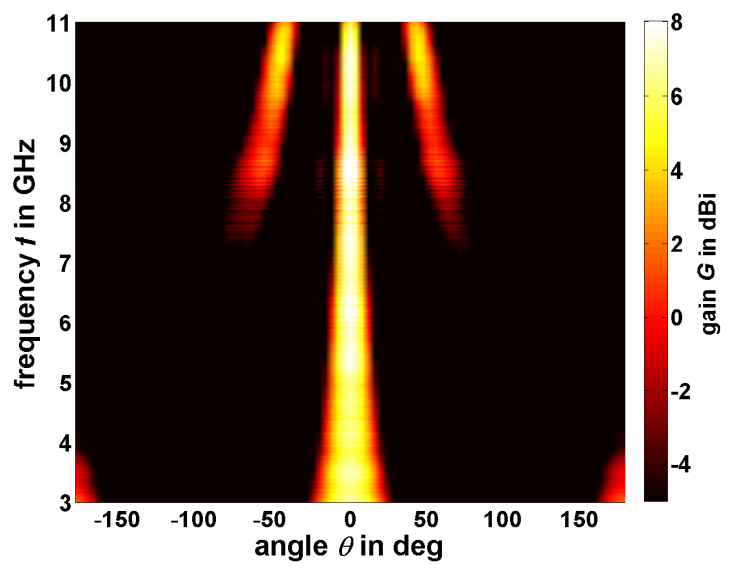

(c) Modeled gain $G(f, \theta)$ of the $4 \times 1$ antenna array in Fig. 3 over angle $\theta$ and frequency $f$.

Fig. 4. Modeled and measured data of the frequency domain parameters.

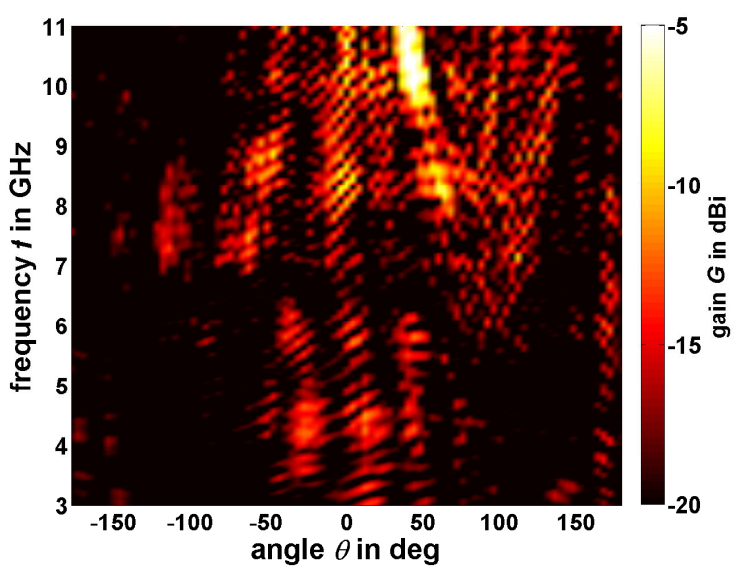

Fig. 5. Measured gain $G(f, \theta)$ (X-Pol) of the $4 \times 1$ antenna array in Fig. 3 over angle $\theta$ and frequency $f$.

is more than $3 \mathrm{dBi}$ more than the mean gain of the single element. From the array theory, by the application of four elements, the gain of the array should be $6 \mathrm{~dB}$ higher than

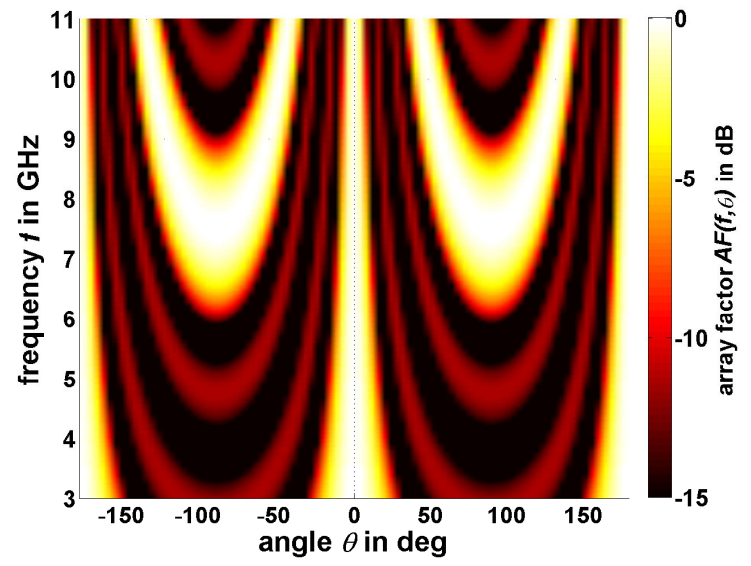

(b) Array factor $A F(f, \theta)$ of the $4 \times 1$ antenna array with distance between the elements of $40 \mathrm{~mm}$ over angle $\theta$ and frequency $f$.

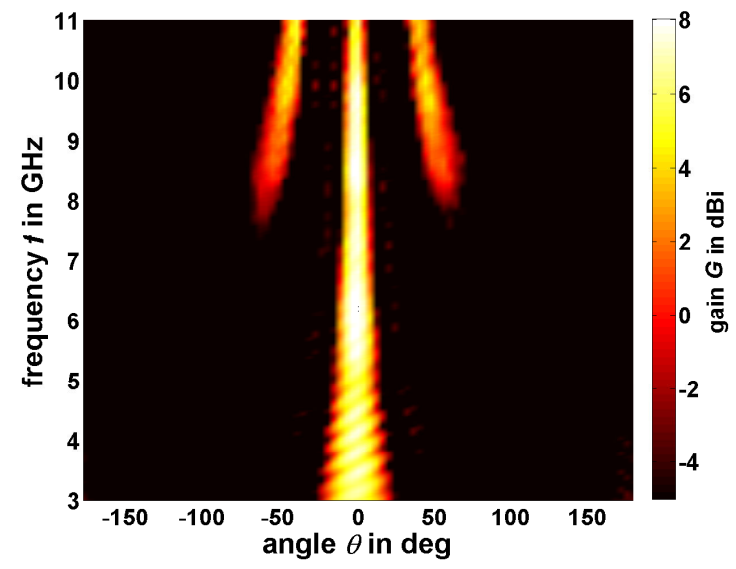

(d) Measured gain $G(f, \theta)$ (Co-Pol) of the $4 \times 1$ antenna array in Fig. 3 over angle $\theta$ and frequency $f$.

of the single element. Here it is not the case because of the additional losses in the feeding network (power divider and $50 \mathrm{~cm}$ long cables). These losses are included in the modeled data.

The side lobes occur near the main lobe and are suppressed by approx. $15 \mathrm{~dB}$. The mean cross-polarized gain in the main beam direction is decoupled from the co-polarized one by more than $20 \mathrm{~dB}$, which is sufficient for an UWB polarimetry.

The additional measurement showed, that the coupling between the elements in the array is in the average lower than $20 \mathrm{~dB}$. This explains a good agreement between the simulated and measured data. Merely for the cross-polarization some differences can be seen. This can be explained by the fact, that in the model all elements are assumed to be exactly the same. In the reality the elements differ slightly from each other.

\section{B. Time domain}

The impulse response can be derived from the frequency domain measurements in the way described in section II. The result obtained for the single array element for vertical polarization (Co-Pol) in the H-Plane is shown in Fig. 7(a). 


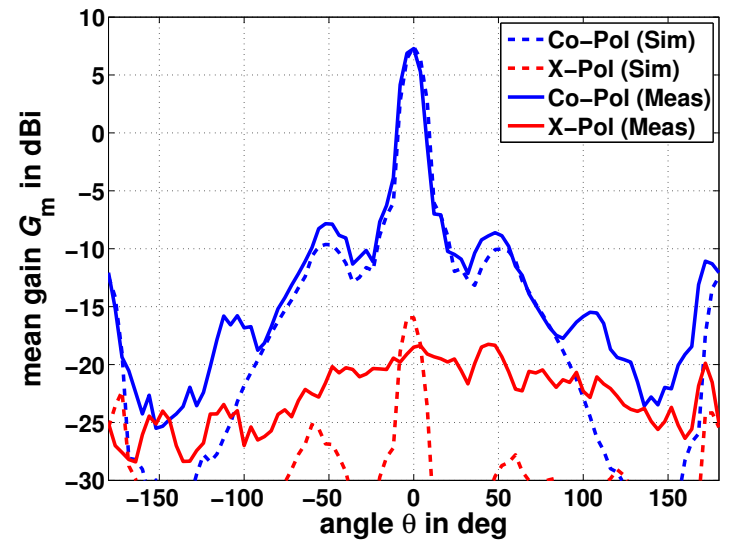

Fig. 6. Mean gain $G_{m}(\theta)$ (Co and X-Pol) of the $4 \times 1$ antenna array in Fig. 3 over angle $\theta$.

The impulse response of the $4 \times 1$ linear array for the same plane calculated accordingly to the model in section III is presented in Fig. 7(b). A significant improvement of the angular resolution can be noticed. The maximum of the impulse response is exactly in direction $0^{\circ}$ and equals $0.32 \mathrm{~m} / \mathrm{ns}$. Aside from the 0 degree direction four paths in each direction can be observed. They come from the radiations from the single antenna elements. It can be noticed that for the linear array with directive array elements there exist only one direction of coherent radiation $\left(0^{\circ}\right)$. In all other angles the pulses from the single array elements can be resolved. It means that for the signals, of which bandwidth is large enough, there is no grating lobes effect. However the increasing distance between the elements causes a spread of the radiated pulse over the time. The spread is maximal for the direction $+/-90^{\circ}$. It can be shortened by the filtering of the array impulse response by the impulse response of the single element. Remarkable is the delay difference caused by the single antenna and the array. The additional delay of approx. $3 \mathrm{~ns}$ is due to the delay of the signal in the feeding network (cf. Fig. 3). The physical length of the feeding network is more than $60 \mathrm{~cm}$. The signal is additionaly delayed by the lower propagation velocity in the medium.

The measured UWB array impulse response is presented in the Fig. 7(c). The amplitude of the measured impulse response differs only slightly from the simulated one. The effect of resolving of the single pulse from the array elements was predicted correctly. Also the additional delay due to the feeding network complies with the measurements.

\section{CONCLUSIONS}

In this paper a dual-orthogonal polarized, linear antenna array consisting of four elements for the IR-UWB applications is described. The gain of the array in the main beam direction is relatively constant and its mean value is $7.2 \mathrm{dBi}$. The mean $3 \mathrm{~dB}$ beamwidth of the main lobe is approx. $15^{\circ}$. The second main lobe and the grating lobes are extensively suppressed by the directive radiation pattern of the single array element. The polarization purity of the signal radiated from the array is very high. The mean decoupling of the polarizations in the far field is higher than $20 \mathrm{~dB}$. The antenna has a very short impulse response, with high angular selectivity. The maximal peak value of the impulse response is $0.32 \mathrm{~m} / \mathrm{ns}$. With its very good performance, the presented dual-polarized UWB antenna array introduces a solution for fully polarimetric IRUWB systems, where a high angular resolution is needed, e.g. Radar.

\section{ACKNOWLEDGMENT}

The authors would like to thank to the German Research Fundation (DFG) for the financial support.

\section{REFERENCES}

[1] Federal Communications Commission (FCC), "Revision of Part 15 of the Commissions Rules Regarding UltraWideband Transmission Systems", First Report and Order, ET Docket 98-153, FCC 02-48; Adopted: February 2002; Released: April 2002

[2] C. A. Balanis, "Antenna Theory: Analysis and Design", Wiley \& Sons, New York, NY, USA, 1996

[3] A.G. Yarovoy, L.P. Ligthar, "UWB Radars for Chalenging Applications", The Third International Conference on Ultrawideband and Ultrashort Impulse Signals, pp. 50 - 55, September 2006

[4] D.L. Sostanovsky,A.O. Boryssenko,E.S. Boryssenko, "UWB radar imaging system with two-element receiving array antenna", 5th International Conference onAntenna Theory and Techniques, pp. 357 - 360, 24-27 May 2005

[5] R. Zetik, J. Sachs, R. Thomae, "UWB Short Range Radar Sensing” IEEE Instrumention and Measurement Magzine, vol. 10, pp. 39 - 45, April 2007

[6] I.A. Osaretin, A. Torres, C. Chen, "A Novel Compact Dual-Linear Polarized UWB Antenna for VHF/UHF Applications", IEEE Antennas and Wireless Propagation Letters, Accepted for future publication, available in IEEXplore

[7] J.-Y. Chung, C. Chen, "Two-Layer Dielectric Rod Antenna”, IEEE Transactions on Antennas and Propagation, vol.56, issue 6, pp. 1541 1547, June 2008

[8] R. C. Paryani, P. F. Wahid, N. Behdad, "A wide-band, dual-polarized, differentially-fed cavity-backed slot antenna", 2008 URSI General Assembly, Chicago, 7-16 August 2008

[9] W. Soergel, S. Knrzer, W. Wiesbeck, "Measurement and Evaluation of U1tra Wideband Antennas for Communications", Proceedings International ITG-Conference on Antennas, pp. 377 - 380, Berlin, September 2003

[10] E. G. Farr, C. E. Baum, "Extending the definitions of antenna gain and radiation pattern into the time domain", Sensor and Simulation Notes, note 350, Directed Energy Directorate, Air Force Research Laboratory, Kirtland, NM, USA, 1992

[11] W. Soergel, W. Wiesbeck, "Influence of the Antennas on the Ultra Wideband Transmission", EURASIP Journal on Applied Signal Processing, special issue UWB - State of the Art, pp. 296 305, March 2005 


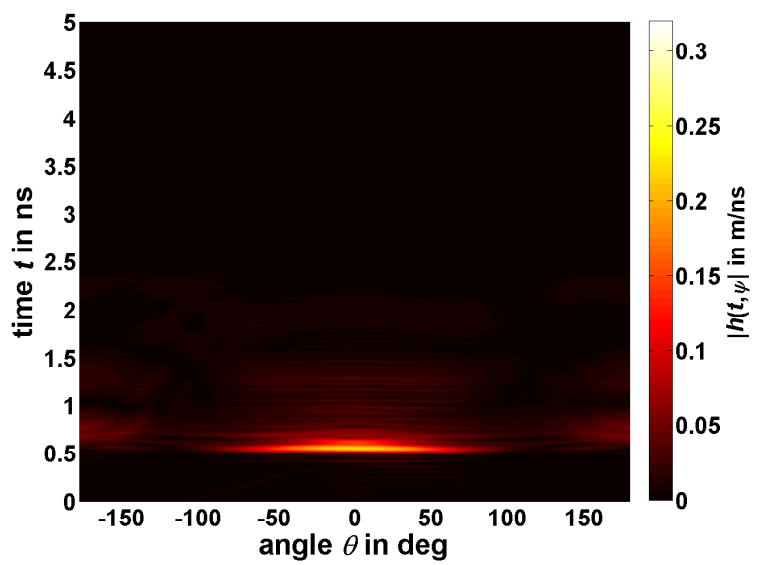

(a) Measured impulse response $h(t, \theta)$ (Co-Pol) of the single array element over angle $\theta$ and time $t$.

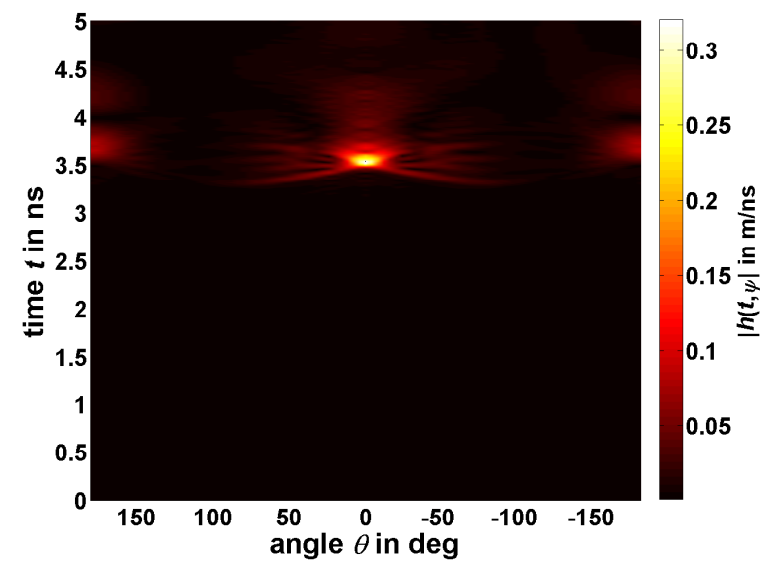

(b) Modeled impulse response $h_{a r}(t, \theta)$ (Co-Pol) of the $4 \times 1$ antenna array in Fig. 3 over angle $\theta$ and time $t$.

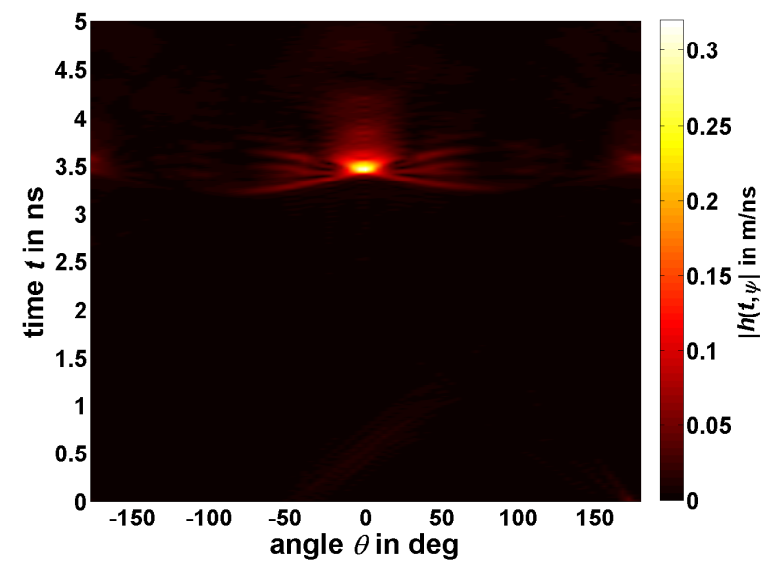

(c) Measured impulse response $h_{a r}(t, \theta)(\mathrm{Co}-\mathrm{Pol})$ of the $4 \times 1$ antenna array in Fig. 3 over angle $\theta$ and time $t$.

Fig. 7. Modeled and measured data of the time domain properties. 